\title{
Diagnosis and management of pancreaticopleural fistula
}

\author{
Clifton Ming $\underline{\text { Tay }}^{1}$, MBBS, Stephen Kin Yong $\underline{\text { Chang }}^{1,2}$, MBBS, FRCSE
}

\begin{abstract}
Pancreaticopleural fistula is a rare diagnosis requiring a high index of clinical suspicion due to the predominant manifestation of thoracic symptoms. The current literature suggests that confirmation of elevated pleural fluid amylase is the most important diagnostic test. Magnetic resonance cholangiopancreatography is the recommended imaging modality to visualise the fistula, as it is superior to both computed tomography and endoscopic retrograde cholangiopancreatography (ERCP) in delineating the tract within the pancreatic region. It is also less invasive than ERCP. While a trial of medical regimen has traditionally been the first-line treatment, failure would result in higher rates of complications. Hence, it is suggested that management strategies be planned based on pancreatic ductal imaging, with patients having poor chances of spontaneous closure undergoing either endoscopic or surgical intervention. We also briefly describe a case of pancreaticopleural fistula in a patient who was treated using a modified Puestow procedure after failed endoscopic treatment.
\end{abstract}

Keywords: ERCP, MRCP, pancreatic fistula, pleural effusion, Puestow procedure

\section{INTRODUCTION}

Pancreaticopleural fistula (PPF) is an uncommon but serious complication of acute, and more commonly chronic, pancreatitis. The precise incidence of PPF is unknown, but it is estimated to occur in $0.4 \%$ of patients with pancreatitis. ${ }^{(1)}$ It falls under the domain of 'internal pancreatic fistulas', a term coined by Cameron et al, and comprises PPF and pancreatic ascites. ${ }^{(2)}$ It refers to the drainage of pancreatic secretions into a body cavity other than the duodenum, secondary to the leakage of pancreatic fluid due to a disruption in a major pancreatic duct (PD). ${ }^{(2)}$ PPF typically presents as massive pleural effusion due to communication between the pancreas and pleural space. The predominance of thoracic symptoms often causes a diagnostic dilemma as initial efforts are directed toward finding a thoracic pathology, thus resulting in a delay in diagnosis. This condition has to be distinguished from the inflammatory effusions associated with acute pancreatitis, which are more common and less severe. ${ }^{(3)}$ Due to the rarity of PPF and the resultant difficulty in conducting controlled prospective trials, there is still ambiguity regarding the diagnostic workup and management of this condition. We review the existing literature to assess the current views on its pathogenesis, presentation, evaluation and management, as well as illustrate certain key points through a brief sharing of our experience in managing a case from our institution.

\section{CASE ILLUSTRATION}

A middle-aged Chinese man with a significant history of recurrent alcoholic pancreatitis presented with worsening shortness of breath for two weeks and unintentional weight loss for over two months. Physical examination and chest radiography detected a massive right pleural effusion. Pleural fluid analysis revealed an exudative pattern. Given the patient's chronic heavy smoking and weight loss, the initial suspicion was that of a malignant pleural effusion. However, subsequent computed tomography (CT) findings showed features of chronic pancreatitis with a dilated proximal PD and foci of calcification in the pancreatic head. This prompted the testing of pleural fluid for amylase levels. A markedly raised pleural fluid amylase level (> 15,000 U/L) confirmed the diagnosis of PPF. Magnetic resonance cholangiopancreatography (MRCP) performed showed a fistula between a dilated side branch of the $\mathrm{PD}$ at the pancreatic tail and a left para-adrenal collection, which extended toward the right pleural space. An initial attempt to stent the main PD via endoscopic retrograde cholangiopancreatography (ERCP) achieved cannulation of the PD, and opacification with contrast showed a tortuous narrow downstream PD with a stone in situ and upstream dilatation. However, stenting failed as the tortuous duct prevented passage of the guide wire. Attempts at endoscopic ultrasonography (EUS) and rendezvous ERCP were then made. Pancreatogram showed a dilated upstream PD with abrupt cutoff at the neck of the pancreas. There was no flow of contrast into the duodenum, but the contrast flowed upstream into a pseudocyst from the ruptured duct at the pancreatic tail. All attempts to pass various wires upstream and downstream failed. Given that all endoscopic treatment options were unsuccessful, the patient underwent a modified Puestow operation. The patient recovered without any complications and remained clinically

${ }^{1}$ Yong Loo Lin School of Medicine, National University of Singapore, ${ }^{2}$ Division of Hepatobiliary and Pancreatic Surgery, Department of Surgery, National University Health System, Singapore

Correspondence: A/Prof Stephen Chang Kin Yong, Senior Consultant, Division of Hepatobiliary and Pancreatic Surgery, Department of Surgery, National University Health System, Tower Block, Level 8,1E Kent Ridge Road, Singapore 119228.cfscky@nus.edu.sg 
well during the outpatient follow-up two weeks postoperatively, with no signs suggesting a recurrence on physical examination.

\section{INCIDENCE AND PATHOGENESIS}

PFF is a rare clinical entity that is estimated to occur in $0.4 \%$ of patients presenting with pancreatitis and $4.5 \%$ of patients with pancreatic pseudocyst. ${ }^{(1)}$ The most common cause of chronic pancreatitis leading to PPF formation is alcohol abuse. Other rarer causes include gallstones, trauma, idiopathic pancreatitis and PD anomalies in paediatric patients. ${ }^{(4)}$ The underlying mechanism of fistula formation involves either a leak or rupture of a pseudocyst, or direct PD disruption. If the leak occurs anteriorly, extrapancreatic communication of the PD with the peritoneum will result in the formation of a pancreaticoperitoneal fistula, which manifests as ascites. If the disruption occurs posteriorly, pancreatic secretions will enter the retroperitoneum and may dissect through the aortic or oesophageal hiatus into the mediastinum. This will either develop into a PPF or mediastinal pseudocyst, which will in turn rupture into the pleural cavity to form a PPF.(4-10) Direct transdiaphragmatic extension of the fistula into the mediastinum has also been reported. ${ }^{(9)}$ The resulting pleural effusion from ascending fistulisation should be distinguished from the small, self-limiting left-sided effusion that occurs in $3 \%-17 \%$ of patients with acute pancreatitis. ${ }^{(3)}$ These reactive effusions are chemically induced or sympathetic in nature, as inflammation of the diaphragm leads to the diffusion of pancreatic enzymes into the pleural space via diaphragmatic lymphatics. ${ }^{(3,7,8)}$ On rare occasions, a fistula may also communicate with the pericardium (pancreaticopericardial), tracheobronchial tree (pancreaticobronchial) or oesophagus (pancreaticoesophageal). ${ }^{(8)}$

\section{CLINICAL FEATURES}

Males in their mid-forties with a history of chronic alcoholism and several previous episodes of pancreatitis form the classical description of patients who develop PPF. ${ }^{(1,9,11,12)}$ In general, PPF formation is seldom associated with acute pancreatitis, and occurs most commonly in a background of chronic recurrent pancreatitis secondary to excessive alcohol intake. ${ }^{(1,4,11,12)}$ The presence of pancreatic pseudocysts was noted in $43 \%-79 \%$ of patients with PPF. ${ }^{(1,5,11,12)}$ There is often a delay in diagnosis of PPF due to the misleading predominance of thoracic symptoms over abdominal symptoms. In the literature, the most common presenting complaint reported was dyspnoea, which was present in $65 \%-76 \%$ of cases. ${ }^{(11,12)}$ Other common presenting complaints include chest pain, cough and abdominal pain, while less common complaints include fever, weight loss and haemoptysis, all of which are often nonspecific. ${ }^{(11-13)}$ Pleural effusions usually occur on the left $(42 \%-67 \%)$, but it is not unusual to find right-sided $(19 \%-40 \%)$ or bilateral $(14 \%-17 \%)$ effusions..$^{(1,9,11-13)}$ The presence of a large, recurrent, rapidly accumulating effusion that is refractory to repeated thoracocentesis in a clinical setting or with a history of pancreatitis should alert one to the suspicion of a PPF.

\section{DIAGNOSIS AND INVESTIGATIONS}

The single most important diagnostic procedure is a pleural tap to determine the level of pleural fluid amylase. There is no established diagnostic threshold for amylase, but pleural fluid amylase would be significantly elevated $(>1,000 \mathrm{U} / \mathrm{L})$, with mean amylase levels above 10,000 U/L (13,000-53,000 U/L) in patients with PPF. ${ }^{(1,11,12,14)}$ Amylase-rich pleural fluid is also seen in conditions such as acute pancreatitis, parapneumonic effusion, pulmonary tuberculosis, oesophageal perforation, liver cirrhosis, hydronephrosis, leukaemia/lymphoma and malignancies of the lung, pancreas, rectum, and in females, the gynaecological system. However, only PPF results in pleural fluid amylase levels above 50,000 U/L..$^{(1,11,13)}$ The pleural fluid protein level would also be high (> $30 \mathrm{~g} / \mathrm{L})$ due to chronic inflammation of the pleural surface. ${ }^{(2,13)}$

Once elevated amylase levels from a diagnostic pleural tap confirms the suspicion of a PPF, several imaging modalities are available for visualisation of the fistula. CT, ERCP and MRCP are most widely used in current practice, and the sensitivity of each modality in detecting PPF is $47 \%, 78 \%$ and $80 \%$, respectively. ${ }^{(11,15)}$ In the past, imaging of PPF was attempted via intrapleural injection of contrast media. ${ }^{(7)}$ At present, although CT of the thorax and abdomen has been successful in identifying PPF, its overall ability to provide accurate delineation of the fistula is disputable and its sensitivity is poor compared to the other modalities. Nevertheless, CT is still useful in demonstrating pancreatic parenchymal atrophy, calcification, duct dilatation and pseudocysts. ${ }^{(8,9)}$ Performing CT immediately after ERCP also increases its sensitivity in detecting the fistula, presumably because of the contrast injected directly in the PD during ERCP.(12)

ERCP used to be the preferred investigation for confirming the diagnosis of PPF. It has the advantage of direct visualisation of the papilla and its adjacent anatomy, thus allowing over-injection of certain ductal portions where fistulae or strictures can occur and the ability to simultaneously perform endoscopic therapeutic manoeuvres. However, ERCP has its shortcomings; it is invasive in nature and unable to clearly demonstrate a fistula if the site of ductal disruption occurs distal to a site of ductal obstruction, such as a stricture. ${ }^{(8,11)}$ Furthermore, the accuracy of ERCP is highly variable (ranging from $0 \%-100 \%$ ), as it is operator-dependent and success is related to the timing of the procedure and the presence of anatomic variations. ${ }^{(16)}$ As such, the use ERCP as a first-line tool for confirmation of PPF is discouraged in view of its limitations and the risk of potentially life-threatening complications such as infection, pancreatitis, bleeding and perforation. ${ }^{(14)}$

Currently, MRCP appears to be the imaging modality of choice due to its noninvasive nature and ability to visualise a fistula beyond strictures. In addition, it is very useful in depicting pancreatic parenchymal and ductal structural changes, as well as the presence of small intra- or extrapancreatic pseudocysts and peripancreatic collections, if any. ${ }^{(8,17)}$ As such, despite its lack of therapeutic options, MRCP is considered the imaging study of choice for PPF due to its superiority over CT in identifying 
a fistula in the pancreatic region and its noninvasiveness as compared to ERCP.(17) Visualising the site of fistulisation and its exact anatomical relationship to the pancreatic ductal tree is particularly useful in the planning of surgical intervention, if surgery is being considered. ${ }^{(4)}$

\section{MANAGEMENT}

The management of PPF can be broadly classified into conservative or surgical. Conservative management consists of medical and endoscopic therapies. The current evidence on conservative management is limited to case reports and case series, and no controlled trials have been conducted due to the infrequency of PPF. Traditionally, a 2-3 week trial of medical treatment is initially attempted, after which failure of resolution or development of complications are considered indications for endoscopic or surgical intervention. ${ }^{(1,14)}$ Given the success of octreotide administration and endoscopic stenting, longer periods of conservative treatment are currently possible treatment with octreotide can be continued for 2.5-6 months and chest drainage for $6-24$ days. ${ }^{(10,18)}$ Medical treatment aims to reduce the stimulation of pancreatic exocrine secretions, and comprises chest drainage, total parenteral nutrition and octreotide administration. ${ }^{(18)}$ Chest tubes should be removed as early as possible, as they can create a pathway of lower resistance for pancreatic secretions distal to a PD obstruction such as a stricture or stone, potentiating the persistence of the fistula. ${ }^{(12)}$ The success rate of managing PPF by medical treatment alone has been reported to be $31 \%-65 \% .^{(1,4,11,12)}$ However, failed medical treatment results in higher rates of complications and prolonged periods of treatment as compared to operative treatment. ${ }^{(4)}$ Therefore, the potential risks of prolonged medical treatment should be weighed against the morbidity and mortality associated with surgery. Appropriate patient selection, based on pancreatic ductal morphology visualised using imaging, might spare 2-3 weeks of futile medical treatment in patients with a poor chance of spontaneous fistula closure. ${ }^{(11,14)}$

Since the first report of successful treatment of PPF by endoscopic stenting of the PD, ${ }^{(19)}$ ERCP has evolved from solely being a diagnostic tool in PPF to a being therapeutic one as well. Restoration of anatomic continuity is more important than reduction of pancreatic secretions by medical treatment alone. ${ }^{(12)}$ Endoscopic stenting has been reported to be an effective therapeutic option with minimal morbidity and mortality, and when used in combination with octreotide, can shorten the period of hospitalisation. ${ }^{(10)}$ ERCP enables the identification of pancreatic duct strictures and stones downstream from the fistula, which can then be treated using pancreatic sphincterotomy, balloon dilatation, stent placement or stone extraction with or without extracorporeal lithotripsy. ${ }^{(20-23)}$ The aim of stent placement is to achieve decompression of the PD by creating a pathway of least resistance for pancreatic secretions to flow into the duodenum. If possible, the stent should also bridge the site of ductal disruption, thus mechanically blocking the fistula lumen, in order to allow healing. ${ }^{(5,19-22)}$ In situations where conventional ERCP and stent placement are difficult due to altered anatomy or difficult cannulation, EUS-guided rendezvous ERCP may be a viable option to obtain antegrade access to the PD. ${ }^{(24-26)}$ This procedure involves puncturing the PD using a linear echoendoscope and a 19-gauge needle via a transgastric approach. A 0.035-inch guidewire is then advanced through the needle into the PD, and downstream across the papilla into the duodenum. With the guidewire in place, the linear echoendoscope is carefully removed and a duodenoscope is passed into the duodenum to grasp the loop of guidewire exiting the papilla. Retrograde cannulation of the PD is achieved by advancing a catheter over the guidewire, and decompression is done via a pancreatic sphincterotomy, followed by transpapillary placement of a plastic stent. ${ }^{(25,26)}$ Endoscopic placement of a nasopancreatic drain with application of low intermittent suction has also been reported to facilitate the resolution of PPF. ${ }^{(12,20-22)}$ This procedure has the added advantage of allowing repeated pancreatograms to confirm fistula closure without the need for ERCP. However, the presence of a tube in the nose is less well-tolerated, and hospitalisation is necessary while the drain is in place due to the high-level nursing care required. ${ }^{(12)}$ The optimal duration for stent or nasopancreatic drainage of PPF is still unknown and can vary from $4-12$ weeks. . $^{(5,14,18,20)}$ It has been suggested that assessing the persistence of the fistula with pancreatograms every six weeks, in order to document the passage of contrast into the pleural space, is a reasonable approach. ${ }^{(10)}$ Nonetheless, there remain concerns of whether longterm stent insertion will induce permanent ductal changes that fail to regress even after stent removal. ${ }^{(18)}$ The present verdict is still inconclusive, given a lack of data on the long-term outcomes of PD stent placement. ${ }^{(5,6,18)}$ It is important to note that stenosis or disruption of the main PD may result in persistent and recurrent fluid collections in a considerable number of endoscopically treated patients. This may, therefore, render the need for surgery in such patients. ${ }^{(18)}$ Similarly, the duration for which endoscopic treatment should be continued before surgical treatment is considered has not yet been clearly established. ${ }^{(18}$

While surgical intervention was considered the first-line treatment for PPF before the era of therapeutic endoscopy, it is now seen as a treatment of last resort, used only after failure of medical and endoscopic therapy. However, in cases with poor chances of spontaneous resolution, delays in definitive surgery with conservative management may result in increased morbidity and mortality. ${ }^{(14)}$ Furthermore, operations performed for PPF are now associated with reasonably low morbidity and mortality rates in high-volume tertiary centres. A review by King et $\mathrm{al}^{(4)}$ observed that the time spent on medical therapy was $50 \%$ more than the time spent on postoperative recovery, indicating that early operative intervention may halve the total duration required for fistula closure. The study has also noted that $70 \%$ of postoperative complications, which included leaks, wound and intra-abdominal infections, recurrence of fistula and development of diabetes mellitus, occurred in patients who converted from 
medical therapy to surgical therapy. ${ }^{(4)}$ As such, primary and early surgical treatment of PPF might prove to be safer, as well as more cost- and time-saving in appropriately selected patients. In fact, the success rate of operative therapy for PPF was more than three times that of medical treatment ( $94 \%$ vs. $31 \%)$, regardless of whether operative therapy was the first-line treatment or performed after failed medical management. ${ }^{(4)}$

To guide treatment strategies, a logical framework may be derived using radiological imaging of the pancreatic ductal anatomy. ${ }^{(11,14)}$ Based on MRCP results, patients with normal or mildly dilated PD and no strictures can be managed with a trial of medical therapy, while patients with ductal disruptions in the pancreatic head or body and a stricture downstream to the disruption should receive therapeutic endoscopy as the firstline treatment. Early surgical intervention is recommended whenever there is complete ductal obstruction, leak in the pancreatic tail, a downstream stricture that cannot be stented, or if the site of ductal disruption cannot be bridged by a stent. ${ }^{(14)}$ Patients who have undergone a failed endoscopic procedure should be treated with surgery as soon as possible, as delays might result in septic complications, such as intra-abdominal abscess and empyema due to superinfection of the pleural or peripancreatic fluid.

The basic principles of surgical treatment for PPF include either some form of pancreatic resection or pancreatic-enteric anastomosis to achieve adequate drainage of secretions. Obstruction of the main PD proximal to the fistula calls for surgical decompression of the obstructed duct, and this may or may not involve resection of the involved portion of the obstructed pancreas. ${ }^{(1,4,5,9,10)}$ The most common operation reported is distal pancreatectomy, followed by pancreaticojejunostomy. ${ }^{(11)}$ In the presence of a pancreatic head mass that compresses the adjacent structures, a Frey procedure, which involves partial resection of the pancreatic head by coring it out, can be performed to decompress both the PD and duodenal/bile duct stenosis. ${ }^{(15)}$ First described by Partington and Rochelle, the modified Puestow procedure is, in brief, a lateral pancreaticojejunostomy that involves side-to-side anastomosis of a longitudinal opening in the jejunum to a longitudinally opened PD. ${ }^{(27)}$ The original Puestow procedure included splenectomy, distal pancreatectomy and longitudinal opening of the main PD with insertion of the pancreas into a Roux-en-Y limb of the jejunostomy, which then acts as a sleeve around the pancreas. ${ }^{(27)}$ The Partington and Rochelle modification obviated the need for removal of the spleen and distal pancreas, and achieved extended drainage of the PD, which was not possible with the original Puestow procedure, where the proximal PD was left undrained because the jejunum could not be brought past the superior mesenteric vessels. ${ }^{(27)}$ One of the key proposed advantages of Puestow and other drainage procedures over resection procedures is the preservation of pancreatic tissue, and hence, exocrine and endocrine functions of the pancreas. ${ }^{(27)}$ This is particularly relevant in patients with chronic pancreatitis who have diminished reserves and may not be able to endure pancreatic resection. However, the benefits of using Puestow and other drainage procedures over resection procedures still remain debatable, as various studies have shown contrasting evidence in terms of the delay in deterioration or improvement of pancreatic function. ${ }^{(27)}$ Nonetheless, there is no evidence suggesting a quickening of dysfunction, as seen in resection procedures. ${ }^{(27)}$

While the modified Puestow procedure is well documented in the literature, its primary indication has been for intractable abdominal pain, and pain control has been used as the main outcome measure of its efficacy in comparison with other approaches. There are no series to date evaluating its use in PPF per se, most likely due to the rare occurrence of PPF. Nevertheless, a consensus statement by the American Gastroenterological Association (AGA) on the use of the modified Puestow procedure in the management of chronic pancreatitis demonstrated morbidity and mortality rates of $0 \%-5 \% .{ }^{(28)}$ Early mortality is mainly due to cardiac and pulmonary complications, while late mortality is usually secondary to the effects of chronic smoking or ongoing alcoholism, such as liver cirrhosis and failure. Early morbidities include postoperative complications such as infection, abscess formation and upper gastrointestinal bleeding, while late morbidities arise from pain recurrence and the risk of missing an existing pancreatic carcinoma that may present years later. ${ }^{(27)}$ Due to the risk of underlying pancreatic malignancy, an intraoperative biopsy of the pancreas should be considered in patients undergoing this operative procedure. ${ }^{(27)}$ In terms of overall outcomes, a review by AGA revealed that the modified Puestow procedure managed to achieve short-term pain relief in $80 \%$ of cases and continued pain relief in $60 \%-70 \%$ of cases two years after surgery. ${ }^{(28)}$ Moreover, subjective improvements in lifestyle, such as increased frequency of return to employment and decreased use of narcotics postoperatively, have been reported. ${ }^{(28)}$

\section{CONCLUSION}

PPF is a rare diagnosis requiring a high index of suspicion due to the predominant manifestation of thoracic symptoms. Pleural fluid analysis, particularly fluid amylase level, is crucial to arriving at the diagnosis. The recommended imaging modality to visualise the fistulous communication is MRCP, as it is superior to both $\mathrm{CT}$ and ERCP in delineating the tract within the pancreatic region, and is less invasive compared to ERCP. MRCP is very useful in depicting the location and extent of a PPF, as well as pancreatic parenchymal and ductal structural changes, which would facilitate the planning of management strategies. Subsequent management plans for PPF depend on the PD morphology seen on MRCP, as it will help to identify patients with poor chances of spontaneous fistula closure on medical therapy alone. Such patients are likely to benefit from either early endoscopic or surgical interventions, depending on the severity of disease, since failed medical treatment tends to result in higher rates of complications and prolonged periods of treatment. As a general rule, only patients with a normal or mildly dilated PD and 
no strictures should be managed with a trial of medical therapy. Those with ductal disruptions or strictures downstream to the disruption should undergo endoscopy, while surgery is warranted in those with complete ductal obstruction, or if stenting does not appear to be possible on imaging.

\section{REFERENCES}

1. Rockey DC, Cello JP. Pancreaticopleural fistula. Report of 7 patients and review of the literature. Medicine (Baltimore) 1990; 69:332-44

2. Cameron JL, Kieffer RS, Anderson WJ, Zuidema GD. Internal pancreatic fistulas: pancreatic ascites and pleural effusions. Ann Surg 1976; 184:587-93.

3. Miller JA, Maldjian P, Seeff J. Pancreaticopleural fistula. An unusual cause of persistent unilateral pleural effusion. Clin Imaging 1998; 22:105-7.

4. King JC, Reber HA, Shiraga S, Hines OJ. Pancreatic-pleural fistula is best managed by early operative intervention. Surgery 2010; 147:154-9.

5. Safadi BY, Marks JM. Pancreatic-pleural fistula: the role of ERCP in diagnosis and treatment. Gastrointest Endosc 2000; 51:213-5.

6. Ridgeway MG, Stabile BE. Surgical management and treatment of pancreatic fistulas. Surg Clin North Am 1996; 76:1159-73.

7. Tombroff M, Loicq A, De Koster JP, Engleholm L, Govaerts JP. Pleural effusion with pancreaticopleural fistula. Br Med J 1973; 1:330-331.

8. Vyas S, Gogoi D, Sinha SK, et al. Pancreaticopleural fistula: an unusual complication of pancreatitis diagnosed with magnetic resonance cholangiopancreatography. JOP 2009; 10:671-3.

9. Sut M, Gray R, Ramachandran M, Diamond T. Pancreaticopleural fistula: a rare complication of ERCP-induced pancreatitis. Ulster Med J 2009; 78:185-6.

10. Dhebri AR, Ferran N. Nonsurgical management of pancreaticopleural fistula. JOP 2005; 6:152-61.

11. Ali T, Srinivasan N, Le V, Chimpiri AR, Tierney WM. Pancreaticopleural fistula. Pancreas 2009; 38:e26-31.

12. Oh YS, Edmundowicz SA, Jonnalagadda SS, Azar RR. Pancreaticopleural fistula: report of two cases and review of the literature. Dig Dis Sci 2006; 51:1-6.

13. Ondrejka $P$, Faller J, Siket $F$, et al. Isolated massive pleural effusion caused by pancreatico-pleural fistula. Z Gastroenterol 2000; 38:583-5.
14. Wronski M, Slodkowski M, Cebulski W, Moronczyk D, Krasnodebski IW. Optimizing management of pancreaticopleural fistulas. World J Gastroenterol 2011; 17:4696-703.

15. Cocieru A, Saldinger PF. Frey procedure for pancreaticopleural fistula. J Gastrointest Surg 2010; 14:929-30.

16. Nordback I, Sand J. The value of the endoscopic pancreatogram in peritoneal or pleural pancreatic fistula. Int Surg 81:184-6.

17. Kiewiet JJ, Moret M, Blok WL, Gerhards MF, de Wit LT. Two Patients with Chronic Pancreatitis Complicated by a Pancreaticopleural Fistula. Case Rep Gastroenterol 2009; 3:36-42.

18. Machado NO. Pancreaticopleural fistula: revisited. Diagn Ther Endosc 2012; 2012:815476.

19. Saeed ZA, Ramirez FC, Hepps KS. Endoscopic stent placement for internal and external pancreatic fistulas. Gastroenterology 1993; 105:1213-7.

20. Hastier P, Rouguier P, Buckley M, et al. Endoscopic treatment of wirsungocysto-pleural fistula. Eur J Gastroenterol Hepatol 1998; 10:527-9.

21. Miyachi A, Kikuyama M, Matsubayashi Y, et al. Successful treatment of pancreaticopleural fistula by nasopancreatic drainage and endoscopic removal of pancreatic duct calculi: a case report. Gastrointest Endosc 2004; 59:454-7.

22. Koshitani T, Uehara Y, Yasu T, et al. Endoscopic management of pancreaticopleural fistulas: a report of three patients. Endoscopy 2006; 38:749-51.

23. Brennan PM, Stefaniak T, Palmer KR, Parks RW. Endoscopic transpapillary stenting of pancreatic duct disruption. Dig Surg 2006; 23:250-4.

24. Mallery S, Matlock J, Freeman ML. EUS-guided rendezvous drainage of obstructed biliary and pancreatic ducts: Report of 6 cases. Gastrointest Endosc 2004; 59:100-7.

25. Cooper ST, Malick J, McGrath K, Slivka A, Sanders MK. EUS-guided rendezvous for the treatment of pancreaticopleural fistula in a patient with chronic pancreatitis and pancreas pseudodivisum. Gastrointest Endosc 2010; 71:652-4.

26. Will U, Meyer F, Manger T, Wanzar I. Endoscopic ultrasound-assisted rendezvous maneuver to achieve pancreatic duct drainage in obstructive chronic pancreatitis. Endoscopy 2005; 37:171-3.

27. O'Neil SJ, Aranha GV. Lateral pancreaticojejunostomy for chronic pancreatitis. World J Surg 2003; 27:1196-202.

28. Ceppa EP, Pappas TN. Modified puestow lateral pancreaticojejunostomy. J Gastrointest Surg 2009; 13:1004-8.

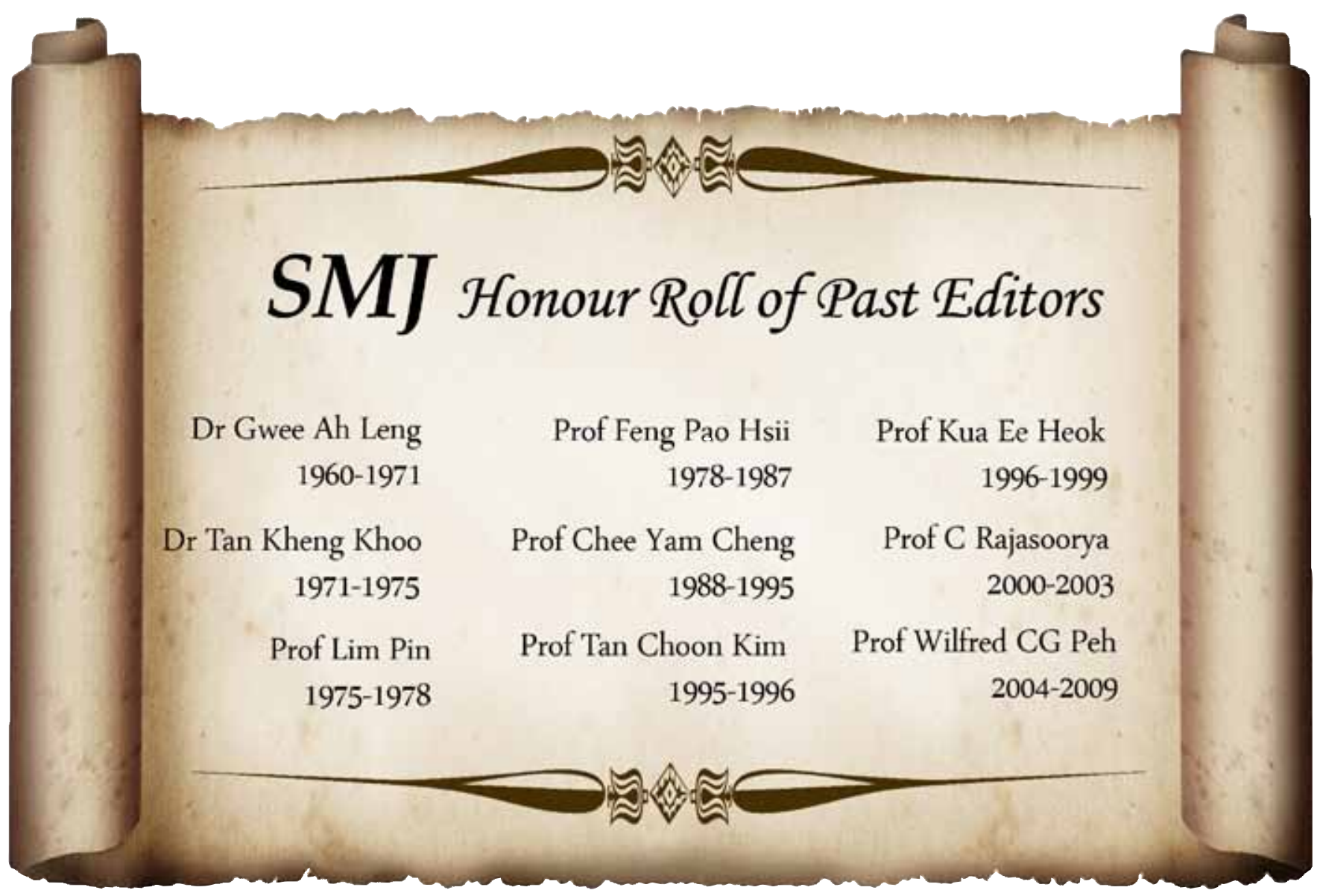

